\title{
Sistema Único de Saúde: uma avaliação realizada em Natal, Rio Grande do Norte, Brasil
}

\author{
Cynthia deFrétas Mdo- UnivesidadeFederal dbRioGrandedbNate, RioGrandedbNate, Brasil \\ JaãoCanlosAlhiei - UnivesidadeFeelarl doRioGrandedbNate, RioGrandedbNate, Brasil \\ JoãoLins deAraújo Neo- Hospital Geal deFataleza, Ceará, Brasil
}

\begin{abstract}
Resumo
O Sistema Único de Saúde (SUS) traduz-se em um conjunto de ações de saúde que deve ser constantemente avaliado. Assim, esta pesquisa objetivou avaliar o SUS em Natal (RN) a partir das crenças de seus gestores, usando uma amostra de nove gestores dos cinco distritos sanitários, que responderam a um roteiro de entrevista semiestruturado, analisados no ALCESTE. Os resultados mostraram que os gestores percebem a importância do SUS na busca da universalização da saúde, reconhecendo, entretanto, que na prática existem barreiras, na atenção básica e no sistema de referência, dificultando o acesso. Problemas que devem-se à má administração dos recursos e ao despreparo dos gestores, indicados por alianças políticas e ausência da comunidade nos Conselhos Municipais de Saúde. Conclui-se que há necessidade de modificar a cultura vigente de que a classe média deve usar planos de saúde, enquanto o SUS destina-se aos pobres.
\end{abstract}

Palavas have Avaliação; Sistema Único de Saúde; G estão em saúde.

\section{Unique Health System: an assessment performed in Natal, Rio Grande do Norte, Brazil}

\begin{abstract}
The Brazilian Unique Health System (SUS) represents a set of health actions that must be constantly evaluated. Thus, this research aimed at evaluating the SUS in Natal, Rio Grande do Norte, Brazil, considering their manager's beliefs, using a sample of nine managers from the five sanitary districts, which answered a semi-structured interview, analyzed through the ALCESTE. The results showed that the managers realize the SUS importance in the search for health universalization, assuming however, the existence of barriers in the basic attention and the reference system, turning the access more difficult. Problems due to resources mismanagement and deficient managers' training pointed out by political alliances, absence of community in the Municipal Health Councils. It is concluded by the need for changing the prevailing culture that the middle class should use private health insurance plans, while SUS is aimed at providing health care to poor people.

Kegnards Assessment; Unique Health System; Health care management.
\end{abstract}

\section{Sistema Ú nico de Salud: una evaluación realizada en Natal, RN-Brasil}

\section{Resumen}

El Sistema Único de Salud (SUS) se traduce en un conjunto de acciones de salud que debe ser constantemente evaluado. Así, esta investigación objetivó evaluar el SUS en Natal (RN)-Brasil partiendo de las creencias de sus gestores, usando una muestra de nueve gestores de los cinco distritos sanitarios, que respondieron un rutero de entrevista semiestructurada, analizados en el ALCESTE. Los resultados mostraron que los gestores perciben la importancia del SUS en la búsqueda de la universalización de la salud, reconociendo, sin embargo, que en la práctica existen barreras, en la atención básica y en el sistema de referencia, dificultando el acceso. Problemas que se deben a la mala administración de los recursos y desarreglo de los gestores, indicados por alianzas políticas, ausencia de la comunidad en los Consejos Municipales de Salud. Se concluye que se necesita modificar la cultura vigente de que la clase media debe usar seguros de salud, mientras el SUS se destina a los pobres.

Palabrasdave Evaluación; Sistema Único de Salud; G estión en salud.

O presente estudo objetivou avaliar o Sistema Único de Saúde (SUS) em Natal, Rio Grande do Norte, a partir das crenças dos seus gestores (diretores e administradores) nos Distritos Sanitários (DS), com o intuito de compreender as condições e os fatores que influenciam o funcionamento efetivo do trabalho de atenção à saúde da comunidade, e identificar os entraves do sistema. Pretendeu-se, dessa forma, fornecer feedbadk às Secretarias Municipais e Estaduais de Saúde, sobre os aspectos positivos e negativos, do SUS.

Para avaliar uma política pública e as ações de programas e estratégias governamentais, faz-se necessário compreender suas diretrizes teóricas, suas propostas e a realidade que se espera encontrar no cotidiano de suas ações. E, em razão da complexidade que envolve a saúde pública e sua organização, esse cuidado torna-se ainda maior.

Legitimado na constituição de 1988 (Brasil, 1988), - Sistema Único de Saúde (SUS) traduz-se no "conjunto de todas as ações e serviços de saúde prestados por órgãos e instituições públicas federais, estaduais e municipais, de administração direta e indireta, e das fundações mantidas pelo Poder Público" (Ministério da Saúde, [MS], 2000, p. 5). Pode contar ainda com as instituições privadas, complementando os serviços de saúde insuficientes ou inexistentes (Albuquerque \& Melo, 2010; Melo, 2007).

Esse conjunto de ações e serviços de saúde funciona integrado numa rede regionalizada $\mathrm{e}$ hierarquizada, num sistema único e organizado por diretrizes que se dividem em princípios doutrinários 
(universalidade, integralidade e equidade) e organizativos (descentralização, hierarquização/ regionalização e participação popular) (Gomes, 2002; Martins, 2008; Melo, 2007; MS, 2000; Negri, 2002; Oliveira, 2006; Polignano, 2001). Considerado um sistema único, porque segue a mesma doutrina e os mesmos princípios organizativos em todo o território nacional, sob a responsabilidade descentralizada das três esferas autônomas de governo: federal, por meio do Ministério da Saúde (MS); estadual, mediante a Secretaria Estadual de Saúde (SES); e municipal, pela Secretaria Municipal de Saúde (SMS) (MS, 2000). Vale salientar que, apesar de seguir os mesmos princípios, a prática cotidiana descortina peculiaridades regionais que diferenciam o sistema ao longo do país, gerando diferenças no funcionamento de suas ações dentro dos três níveis de atuação.

Três níveis que devem funcionar de forma integrada, possuindo ações diferenciadas e bem definidas: 1) Atenção Básica, porta de entrada do SUS, referente à atenção prestada por profissionais generalistas, mais voltados à promoção de saúde, prevenção de doenças e à resolutividade dos problemas menos graves, realizada nas Unidades Básicas de Saúde (UBS) e, principalmente, na Estratégia Saúde da Família, dentro das Unidades de Saúde da Família (ESF); 2) Atenção Secundária, que envolve ambulatórios e tecnologia de média complexidade na realização de exames e consultas com especialistas (pediatras, oftalmologistas, psiquiatras, dermatologistas, cardiologistas, otorrinolaringologistas, entre outros) nos centros de referência; e 3) Atenção Terciária, que se refere aos procedimentos correspondentes a hospitais e tecnologias de alta complexidade (Gomes, 2002; Melo, 2007; O liveira, 2006).

Desse modo, através do SUS, toda a população deve ter acesso à saúde, sendo atendida na Atenção Básica e sendo referenciada para os níveis mais complexos de atendimento, quando necessário. Assim, a saúde se torna um direito universal, independentemente de sexo, raça ou crença; cabendo ao SUS priorizar promoção da saúde àqueles que mais necessitam, de modo a gerar equidade no acesso à saúde; sempre visando 0 ser humano em sua totalidade, reconhecendo que cada pessoa é um ser indivisível e integrante de uma comunidade, para que as ações de saúde sejam realizadas num conjunto integrado e em equipe transdisciplinar. $\mathrm{E}$ para que 0 sistema funcione na prática, sua organização deve respaldar-se numa gestão onde as responsabilidades sejam redistribuídas entre os vários níveis de governo, de modo que os gestores possam estar mais próximos dos serviços; que devem funcionar respeitando a hierarquização do sistema, onde os três níveis (A tenção
Basica, Secundaria e 'l'erciaria) devem funcionar seguindo sua hierarquia e de forma interligada, por meio da referência e contrarreferência; reforçando ainda a importância dos Conselhos Municipais de Saúde e cultivando a participação da população neles, para uma efetivação do controle social e da participação popular (Melo, 2009; Polignano, 2001).

Respeitando também as peculiaridades locais desse sistema que, apesar de único, diferencia-se pelas modificações que sofre durante sua prática cotidiana, faz-se necessário conhecer a organização da gestão em Natal, RN. Desde 1998, pela Norma Operacional Básica 96, a cidade está habilitada na gestão plena do sistema municipal de saúde, possuindo, portanto, todos os três níveis de atenção de saúde. Em virtude da amplitude do sistema na capital, sua gestão é distribuída por divisão territorial de cinco Distritos Sanitários (D S): D S. Sul, D S. O este, D S. Leste, D S. Norte I e DS. Norte II. Esses polos possibilitam a regionalização da gestão, que é realizada mais próxima dos centros de saúde, administrando e coordenando as ações prescritas pela Secretaria Municipal de Saúde, funcionando como elo entre esta e as UBSs, USFs, centros e clínicas especializadas da atenção secundária e hospitais da atenção terciária (Ministério da Saúde, [MS], 2001; Ministério da Saúde, [MS], 2004; Ministério da Saúde, [MS], 2006). Contemplando ainda que a cidade conta com 37 USFs, em 116 equipes, que cobrem 95\% da população, num total de 95\%, 251.850 pessoas (Melo, 2011).

É um sistema amplo e complexo, que necessita ser constantemente avaliado. Não apenas a partir das queixas de seus usuários e profissionais, como fazem a maioria das pesquisas de avaliação do SUS, mas buscando também contemplar os gestores, pois são eles que reconhecem a realidade do sistema no seu aspecto burocrático e financeiro e no que diz respeito às questões ligadas mais diretamente aos seus formuladores (Ministério da Saúde e Secretaria Municipal de Saúde). São eles que reconhecem as limitações do sistema antes mesmo de aparecerem as dificuldades dos profissionais e as queixas dos usuários. Por meio deles é possível visualizar o que está por trás da ponta do icberg(Albuquerque, Melo, Souza Filho \& Araújo Neto, 2011; Belloni, Magalhães \& Sousa, 2003; Martins, 2008; Melo, 2011).

Não existe no Brasil uma forte cultura de avaliação de políticas sociais, para verificação dos resultados dos investimentos realizados com dinheiro público (Lobo, 2001). Reconhece-se, entretanto, que 0 psicólogo seria indicado para tal tarefa, já que o mesmo é preparado profissionalmente para construção de instrumentos de avaliação de comportamento, mediante de um extenso arcabouço teórico: relações 
intergrupais, crenças coletivas, atitudes, diferenças entre gêneros, comportamentos individuais e grupais (Albuquerque, 1999).

D estacam-se, dentre estas, a ênfase, na presente pesquisa, à teoria das crenças da psicologia social e teoria cognitivo-comportamental. Tais teorias reconhecem que a cognição representa a síntese de estímulos internos e externos e evidencia 0 modo como a pessoa avalia uma situação. Refletem a visão da pessoa sobre si mesma, seu mundo, seu passado e seu futuro. E as alterações no seu conteúdo repercutem sobre o estado afetivo e o padrão comportamental (Cordioli, 2008). Assim, o estudo das crenças em psicologia social torna-se um tema clássico e relevante, principalmente quando se acrescenta a suposição de que essas crenças produzem consequências na cognição, na afetividade, na conduta, no comportamento e na personalidade dos indivíduos (Krüger, 2004).

D essa forma, compreende-se que os gestores possuem crenças sobre o SUS, assertivas, que são adquiridas no contato direto com objeto de crença, no seu cotidiano de trabalho, de gestão e operacionalização do sistema. Crenças essas que repercutem sobre 0 estado afetivo e padrão comportamental do sujeito ante o sistema (Cordioli, 2008; Rokeach; 1981), e embora se tratem de variáveis intervenientes, podem ser inferidas e integrar hipóteses empíricas, pois são acessíveis à mensuração, ainda que de forma indireta (K rüger, 2004).

Não se buscou na presente pesquisa, portanto, meramente apontar defeitos, mas, conforme sugere a literatura (Belloni \& cols., 2003; Silva \& Formigli, 1994; Tanaka \& Melo, 2000; Tripodi, Fellin \& Epstein, 1975), objetivou-se avaliar o SUS em Natal a partir das crenças dos gestores (diretores e administradores) dos Distritos Sanitários (DS), com 0 intuito de compreender as condições e os fatores que influenciam o funcionamento efetivo do trabalho de atenção à saúde da comunidade, como forma de proporcionar um feedbadk que possa efetivamente auxiliar nas decisões dos gestores, na busca do aprimoramento dos serviços de saúde prestados à população.

\section{Método}

\section{Tipodepesquisa}

O presente estudo trata-se de uma pesquisa descritiva, de caráter exploratório, que tem como objetivo explorar e descrever essa temática sob a ótica dos gestores, contribuindo com essa temática pouco investigada e escassa na literatura, considerando os aspectos qualitativos do discurso dos participantes.

\section{Participantes}

Foi utilizada uma amostragem em consenso com todos os nove participantes: cinco diretores dos cinco Distritos Sanitários da cidade (DS Sul, DS Leste, D S Oeste, DS Norte I e DS Norte II) e pelos quatro administradores atuantes no DS Leste, D S O este, DS Norte I e DS Norte II, não contando-se com 0 administrador do DS Sul, pela falta desse no quadro de funcionários. Estes foram enumerados aleatoriamente de um a oito, sendo assim chamados, sem identificalos.

\section{Instrumentos}

Como instrumentos para coletar os dados desta pesquisa foi utilizado um roteiro de entrevista semiestruturada, abordando as seguintes categorias: 1) compreensão sobre o sistema de saúde na capital; 2) avaliação do sistema de saúde da capital; 3) operacionalização dos distritos sanitários; 4) compreensão e avaliação do funcionamento do Conselho Municipal de Saúde; 5) sistema de referência e contrarreferência.

\section{Procedimentos éicos edecdła dedados}

Considerando-se os aspectos éticos referentes a pesquisas envolvendo seres humanos, 0 estudo foi aprovado pela Secretaria Municipal de Saúde e pela Comissão de Ética em Pesquisa do Hospital Universitário Onofre Lopes (CEP/HUOL), sob 0 protocolo de $n^{\circ}$ 0298, em 09 de julho de 2008. Foram, então, realizadas visitas aos Distritos Sanitánios de Saúde, onde os participantes foram informados previamente dos objetivos e procedimentos do estudo, sendo solicitado, ainda, que os participantes assinassem um Termo de Consentimento Livre e Esclarecido. Os instrumentos foram então aplicados de forma individual, com uso de gravador, garantindo 0 anonimato da sua colaboração e a confidencialidade de suas respostas.

\section{Análisededadbs}

Para a análise de dados aferidos realizou-se uma análise lexical mecanizada das entrevistas, com auxilio do saftuareAlceste 4.5 (AnalyseLexicalepar Contextedum Ensenble de Segments de Texte, que permite realizar análise de conteúdo por meio de técnicas qualiquantitativas de tratamento de dados textuais e se propõe a identificar a informação essencial presente em um texto. D essa maneira, a partir do Alceste, recorreuse à análise das co-ocorrências das palavras nos enunciados que constituem o texto, para organizar e sumariar informações consideradas mais relevantes, possuindo como referência, em sua base metodológica, a abordagem conceitual e dos "mundos lexicais". 
Gerando, deste modo, categorização mecânica dos dados a partir das Unidades de Conteúdo Elementar (UCE), distribuídas em classes temáticas.

\section{Resultados}

Este tópico aborda as análises realizadas com 0 intuito de identificar as crenças dos representantes municipais da saúde sobre o Sistema Único de Saúde (SUS), a Estratégia Saúde da Família (ESF), como sua porta principal, por meio da Atenção Básica e da integração dela com os demais níveis de atenção, e sobre o funcionamento gestor e fiscalizador do sistema, além de verificar as inter-relações existentes entre os contextos temáticos derivados do coms dos representantes municipais da saúde. 0 conus geral foi constituído por nove entrevistas, ou seja, Unidades de Contexto Inicial (UCI), totalizando 25.269 ocorrências (palavras, formas ou vocábulos), sendo 2.919 palavras distintas, com uma média de nove ocorrências por palavra. Para a análise que se seguiu foram consideradas as palavras com frequência igual ou superior à média de três e com quiquadrado superior a $3,84\left(\chi^{2}>3,84\right)$, visto que só se considera significativo $\chi^{2}$ acima desse valor e com 1 grau de liberdade. Após a redução dos vocabulánios às suas raízes, foram encontrados 543 radicais e 899 unidades de contexto elementar (UCE). Seguindo, então, esse critério, das 899 UCEs, a classificação hierárquica descendente $(\mathrm{CDH})$ reteve 638 que seguiram sendo analisadas, correspondendo a $71 \%$ do conps ou seja, após a redução dos vocábulos às suas raízes lexicais, foram eliminadas apenas 29,03\%. Estas UCEs analisadas foram distribuídas em três classes, formadas com, no mínimo, 10 UCEs (classe 1, com 64,73\% das UCEs; classe 2, com 23,04\%; e classe 3, com 12,23\%).

Destaca-se que essas três classes encontram-se divididas em duas ramificações (A e B) do compus total em análise. 0 subcomus A, composto pela classe 1 (organização e funcionamento do Sistema Único de Saúde), foi denominado "Organização e funcionamento do Sistema Único de Saúde" (com distância euclidiana 9,8 e grau de similitude/ proximidade $\mathrm{r}=0,02$ ), e refere-se às crenças sobre o SUS; e o subcopus B, contendo os discursos correspondentes às classes 2 (gestão e fiscalização da Estratégia Saúde da Família) e 3 (recursos humanos e materiais da Estratégia Saúde da Família), foi denominado "Organização e funcionamento da Estratégia Saúde da Família" (com distância euclidiana 7,8 e grau de similitude/ proximidade $r=0,22$ ), que contempla as crenças sobre os serviços da atenção básica da ESF. A seguir serão descritas, operacionalizadas e exemplificadas cada uma dessas classes.

\section{Classe I - Organização eFunianamento do Sistema Único de Saúde}

A classe I foi constituída por 130 UCE, representando 64,73\% do copus total analisado. Essa classe é composta por palavras e radicais no intervalo entre $\chi^{2}=27$ (Sistema Único de Saúde) e $\chi^{2}=8$ (marct, méd+, vidat, plano de saúde, volt+, aspecto+). De acordo com as variáveis descritivas dos dados biodemográficos, predominaram UCEs derivadas do discurso dos diretores (304 UCE; $\chi^{2}=8$ ); dos entrevistados que conhecem o Conselho Municipal de Saúde - CMS (340 UCE; $\chi^{2}=26$ ); que atribuíram a menor nota aos instrumentos e equipamentos da ESF (220 UCE; $\left.\chi^{2}=21\right)$. Quanto às raízes lexicais que obtiveram maior associação nesta classe, destaca-se: sistema único_de saúde (Sistema Único de Saúde); estratégia_saúd̄e_da_família (Estratégia Saúde da Família); atenção básica (atenção básica); atenção_terciária (atenção terciária); referenci+ (referência, referenciar, referenciado), exam+ (exame, exames), marc+ (marcação, marcando, marcar); volt+ (volta, voltada, voltado, voltando, voltar, volte); paciente+ (paciente, pacientes); população; plano de saúde (plano de saúde); med+ (médico, médicos); vida+ (vida, vidas); aspcto+ (aspecto, aspectos).

Essa primeira classe divide-se em seis subclasses: 1.1 Os três níveis de atenção à saúde no SUS (1.1.1 Atribuição da responsabilidade operacional dos três níveis de atenção: atenção básica, atenção secundária, atenção terciária; 1.1.2 Atribuição da responsabilidade gestora dos três níveis de atenção: município, estado e federação); 1.2 Aplicação de recursos financeiros do SUS (1.2.1 Forma de aplicação dos recursos financeiros; 1.2.2 Desvio de recursos financeiros); 1.3 Avaliação do SUS e da ESF (1.3.1 Avaliação do SUS: aspectos positivos do SUS, aspectos negativos do SUS; 1.3.2 Avaliação da ESF: aspectos positivos da ESF, aspectos negativos da ESF); $1.4 \mathrm{O}$ SUS e a população (1.4.1 Vínculo do SUS com a comunidade; 1.4.2 Público alvo: SUS para todos, SUS para os pobres; 1.4.3 Os menos esclarecidos; 1.4.4 Os formadores de opinião); 1.5 Acessibilidade aos serviços (1.5.1 Atendimento no nível básico: possibilidades no atendimento, entraves no atendimento; 1.5.2 Atendimento no nível secundário e terciário: possibilidades no atendimento e entraves no atendimento); e 1.6 Sistema de referência (1.6.1 Compreensão dos gestores sobre o objetivo do sistema de referência; 1.6.2 Possibilidades da referência; 1.6.3 
Entraves da referência; 1.6.4 Soluções para a referência; 1.6.5 Necessidade da contrarreferência).

$\mathrm{Na}$ análise realizada, verificou-se que nessas subclasses estão contemplados os discursos dos gestores que enfatizam a organização geral do Sistema Unico de Saúde, destacando-se a distribuição de responsabilidades operacional e gestora dos seus três níveis de atenção (atenção básica, secundária e terciária); a aplicação de seus recursos financeiros; a avaliação que os gestores fazem do SUS e da ESF, destacando seus aspectos positivos e negativos; a relação do SUS com a população; a avaliação da acessibilidade aos serviços, tanto no nível de atendimento básico quanto na marcação de exames e especialistas no nível secundário e terciário; destacando ainda 0 sistema de referência, na forma do processo de encaminhamento de pacientes da atenção básica, nas ESFs, aos serviços de média e alta complexidade (sistema de referência), bem como ao retorno de pacientes dos serviços de alta e média complexidade à atenção básica (contrarreferência), na forma de um laudo ou formulário registrando o seu estado de saúde. Dentro desse conteúdo categorizado, seguindo a sequência de cada subclasse, podem-se caracterizar as crenças expressadas e compartilhadas pelos gestores (diretores e administradores) quanto à organização e ao funcionamento do Sistema Unico de Saúde em:

1. O SUS está distribuído em três níveis de atenção, sendo o município responsável pela atenção básica e o Estado pela atenção secundária e terciária. Destaca-se a tendência de que futuramente seja repassada, gradualmente, a atenção secundária para a responsabilidade do município.

2. Os recursos financeiros destinados à saúde não são aplicados de forma correta. Enfatiza-se a necessidade de priorizar o investimento na atenção básica e não nos hospitais. Destacam-se ainda as suspeitas de desvio de verba.

3. O SUS e a ESF são modelos internacionais de atenção à saúde e contribuem para proporcionar atenção à saúde à população. Possuem, entretanto, entraves que dificultam sua operacionalização.

4. A população possui um vínculo direto com 0 SUS, destacando-se, entretanto, que a falta de esclarecimento dos menos favorecidos economicamente dificulta a luta por seus direitos. Contempla-se ainda que a integração da classe média ao sistema, ao invés do uso de planos de saúde, pode contribuir para a melhora dos serviços.

5. O SUS e a ESF oferecem possibilidades de acesso à população, apesar de possuir entraves que dificultam sua acessibilidade, desde a ausência de profissionais nas Equipes de Saúde da Família, até os problemas com o Sistema de Regulação do SUS
(SISREG) que encaminha referência e contrarreferência de pacientes da atenção básica para demais níveis.

6. 0 sistema de referência serve para a triagem e 0 encaminhamento dos usuários da atenção básica para os níveis de maior complexidade. É dificultado, entretanto, pela limitação da quantidade de médicos especialistas e vagas de exames que retardam 0 referenciamento, sendo sugerido como alternativo recorrer ao setor privado, na complementação desses serviços.

\section{Classe II - Gestão e Fiscalização da Estratégia Saúde da Familia}

Essa classe foi constituída por 67 UCE, representando $23,04 \%$ do copus total analisado, composta por palavras e radicais no intervalo entre $\chi^{2}=201$ (Conselho Municipal de Saúde) e $\chi^{2}=16$ (integração, funç+). Predominaram UCEs derivadas do discurso do gestor 8 , que foi o que apresentou maior nota para 0 sistema de referência $\left(14 \mathrm{UCE} ; \chi^{2}=18\right)$. As principais raízes lexicais que obtiveram maior associação nesta classe corresponderam a: conselho_municipal_de_saúde (Conselho Municipal de Saúde); secretaria municipal_de_saúde (Secretaria Municipal de Saúde); reuni+ (reunião, reuniões); gest+ (gestão); particip+ (participa, participação, participantes, participei, participo); distrito+ (distrito, distritos), trabalhador+ (trabalhador, trabalhadores); gestor+ (gestor, gestora, gestores); nome+ (nome, nomeação, nomeada, nomeia); secretarit (secretaria, secretárias, secretário); respond+ (responder, respondeu); form+ (forma, formação, formado); cargo+ (cargo, cargos); sindicato+ (sindicato, sindicatos); assum+ (assumir, assumiu); integração; func+ (função, funções).

A classe II divide-se em quatro subclasses: 2.1 Conselho Municipal de Saúde - CMS (2.1.1 Composição do CMS; 2.1.2 Participação no CMS; 2.1.3 Funcionamento do CMS; 2.1.4 Função do CMS; 2.1.5 Pontos positivos do CMS; 2.1.6 Pontos negativos do CMS); 2.2 Gestão em ESF (2.2.1 Compreensão sobre gestão em ESF; 2.2.2 Necessidades na gestão em ESF); 2.3 Integração com a Secretaria Municipal de Saúde; e 2.4 Forma de ocupação do cargo de gestor.

Desse modo, observa-se que esta classe diz respeito a gestão e a fiscalização da Estratégia Saúde da Família, verificando-se a organização geral do Conselho Municipal de Saúde (CMS), contemplando sua composição, a participação dos gestores entrevistados, seu funcionamento, função, bem como seus pontos positivos e negativos. D estacou-se também a compreensão e as necessidades da gestão em ESF. Pontuou-se ainda o discurso com as crenças dos 
gestores sobre a integraçao dos distritos sanitarios com a Secretaria Municipal de Saúde. E, por fim, elencaramse as formas de ocupação do cargo de gestor.

Pode-se, a partir das análises dessa classe, apurar que as crenças compartilhadas pelos gestores quanto à gestão e fiscalização da Estratégia Saúde da Família foram:

1. O CMS é um órgão fiscalizador de extrema importância, que defende os direitos da população, mas que, entretanto, tem agido apenas com criticas, e não como colaborador.

2. A gestão da ESF, embora ainda pouco compreendida pelos gestores, é realizada pela coparticipação dos trabalhadores, usuários e gestores.

3. A integração dos distritos sanitários com a SMS é imprescindível e realizada por meio de reuniões periódicas.

4. Os cargos de gestor são realizados por indicação política.

\section{Classe III - Reursos Humanos e Mateiais da Estratéja Saúdeda Familia}

E por fim, a classe 3 foi constituída por $71 \mathrm{UCE}$, representando 12,23\% do copus total analisado, composta por palavras e radicais no intervalo entre $\chi^{2}=102$ (capacitações) e $\chi^{2}=22$ (reform+, piores, localização, sonh+, todas). Não se destacou nenhuma variável descritiva, as principais raízes lexicais que obtiveram maior associação nesta classe corresponderam a: capacitações; unidade_de_saúde_da_família (Unidade de Saúde da Família); capacit+ (capacita, capacitação, capacitado, capacitando, capacitar) estrutura fisica (estrutura física); equipamento+ (equipamento, equipamentos); odontológ+ (odontologia, odontológica, odontológico, odontológicos) manutenção; prédio+ (prédio, prédios), acessibilidade; instrumentos; recursos humanos (recursos humanos); boat (boa, boas); propr+ (própria, próprios); precar+ (precária, precário, precários); ruim; quantidade; bons; reform + (reforma, reformando), piores, localização, sonh+ (sonho), todas.

Os conteúdos se distribuíram em duas subclasses: 3.1 Recursos materiais da Estratégia Saúde da Família (2.1.1 Equipamentos e instrumentos das Unidades de Saúde da Família; 2.1.2 Infraestrutura física das Unidades de Saúde da Família) e 3.2 Recursos humanos da Estratégia Saúde da Família (3.2.1 Capacitações dos recursos humanos; 3.2.2 Insuficiência de recursos humanos), onde foram descritos e avaliados os recursos humanos e materiais disponíveis na Estratégia Saúde da Família. Assim, esta classe representa a realidade da quantidade e qualidade dos equipamentos e instrumentos disponíveis nas Unidades de Saúde da Família. Foi contemplada ainda a avaliação da disponibilidade, qualidade e entraves das capacitações oferecidas pela Secretaria Municipal de Saúde aos profissionais, bem como a insuficiência destes na composição das equipes de Saúde da Família. Sintetizando, as crenças, de forma geral, derivadas dessa classe foram:

1. Os materiais odontológicos têm sido trocados nas Unidades de Saúde da Família (USF's), mas o restante dos materiais e equipamentos está defasado e sua manutenção é retardada devido a burocracias internas.

2. As Unidades de Saúde da Família funcionam, em sua maioria, em casas alugadas que foram adaptadas, mas que não possuem infraestrutura adequada para 0 funcionamento da Estratégia Saúde da Família.

3. A Secretaria Municipal de Saúde oferece capacitações em número satisfatório. Estas, entretanto, são feitas sem planejamento de datas e temas de interesse. Como consequência, aliado a outros fatores, os profissionais não demonstram interesse em participar dos cursos ou serem agentes multiplicadores do conhecimento.

4. A Estratégia Saúde da Família ainda sofre com a insuficiência na quantidade e qualidade dos profissionais que compõem as Equipes de Saúde da Família.

\section{Análisefatorial por comespondância}

Além dos resultados obtidos por meio da Classificação Descendente Hierárquica (CDH), realizou-se a Análise Fatorial por Correspondência (AFC), resultando no plano fatorial demonstrado na Figura 1. A AFC permitiu a visualização das diferenciações grupais no plano fatorial, evidenciando as suas especificidades, avaliando de que modo estas classes de significados e seus conteúdos mais característicos se relacionam entre si. Tal figura demonstra 0 traçado de dois eixos, que juntos explicaram $100 \%$ da variância total das UCEs, contendo as oposições entre as três classes emergentes. É possível visualizar a projeção das variáveis suplementares, bem como o conteúdo da fala dos gestores que se distribuiu em três zonas ou conglomerados, de modo não aleatório e correspondente às formas específicas das classes.

No eixo 1, na linha horizontal, à direita, concentra-se a Classe 1 (O rganização e funcionamento do Sistema Único de Saúde), que explica $54 \%$ da variância total das UCEs. E no eixo 2, na linha vertical, superior, destaca-se a classe 2 (G estão e fiscalização da Estratégia Saúde da Família). E no lado inferior do mesmo eixo, por oposição, posicionam-se as palavras da classe 3 (Recursos humanos e materiais da 
Estratégia Saúde da Família). Juntas, explicam 46\% da variância total das UCE.

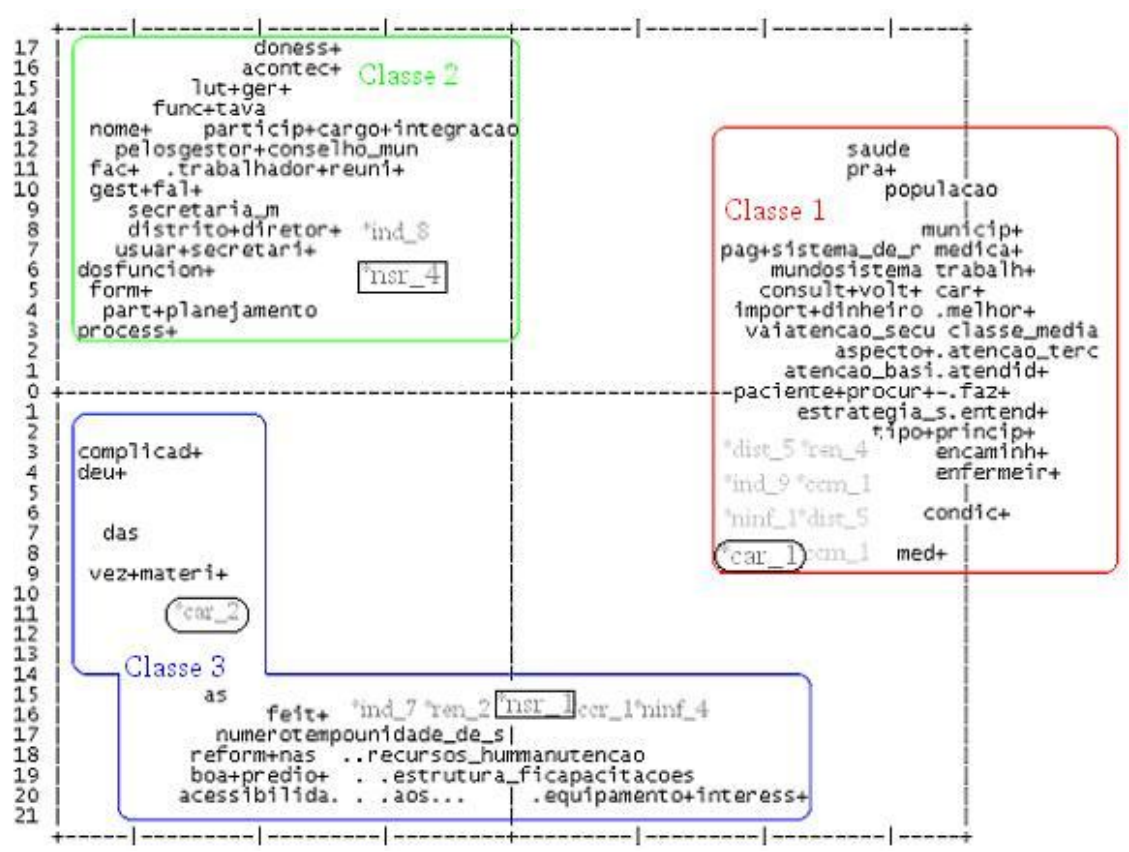

Figura 1. Plano fatorial: projeção dos conteúdos mais significativos sobre o Sistema Único de Saúde

Desse modo, pela análise do plano fatorial, verifica-se que foram extraídas duas grandes dimensões acerca das crenças dos gestores sobre os serviços públicos de saúde. A primeira dimensão está voltada para as crenças sobre a organização e o funcionamento do Sistema Único de Saúde, avaliando a realidade do sistema maior, destacando os serviços de seus níveis de atenção à saúde e a integração destes, que reflete sobre o sistema de referência. Nessa dimensão destacam-se as crenças dos gestores que ocupam o cargo de diretores dos distritos sanitários.

A segunda dimensão reflete as crenças sobre a organização e funcionamento da Estratégia Saúde da Família. Caracterizam-se pela composição de duas classes, distintas, em oposição, mas que, juntas, formam a avaliação global sobre a estratégia, que, por um lado, perpassa as crenças sobre a parte gestora e fiscalizadora (classe 2), no topo da pirâmide do sistema; e, por outro lado, chega às crenças sobre os recursos materiais e humanos na parte operacional do cotidiano da prestação de serviços nas Unidades de Saúde da Família (classe 3), na base da pirâmide do sistema. Destaca-se que essas duas aglomerações se encontram em oposição também pela variável "ncr" (nota atribuída ao sistema de referência): na parte superior (classe 2), salienta-se as crenças dos gestores que melhor avaliaram o sistema de referência; e na parte inferior (classe 3), estão as crenças dos gestores que pior avaliaram o sistema de referência. Pode-se inferir que, por um lado, a ênfase na importância da integração com a SMS e o envolvimento do CMS reflete uma boa avaliação da integração dos três níveis no sistema de referência. E, por outro lado, as crenças sobre as dificuldades de recursos humanos e materiais, desde a atenção básica, refletem uma descrença sobre 0 funcionamento dos demais níveis.

Vale salientar, ainda, que no aglomerado da classe 2 predominam as crenças dos gestores que ocupam 0 cargo de administrador do distrito. Estes são os gestores que possuem o trabalho mais direto com os diretores das Unidades de Saúde da Família, vivenciando mais diretamente a realidade destas. 0 que justifica 0 fato de ser nesses aglomerados onde se concentram as crenças relacionadas à realidade operacional das unidades, de condições de trabalho, no que diz respeito aos recursos materiais (equipamentos e instrumentos, e infraestrutura física) e recursos humanos (capacitações e insuficiência de funcionários). Encontra-se em oposição ao aglomerado da classe 1, onde predominam as crenças dos diretores do distrito, que trabalham não apenas com a Estratégia Saúde da Família, mas com toda a rede do SUS, o que justifica 0 fato de seus discursos serem mais abrangentes, contemplando crenças sobre todo o sistema. 


\section{Discussão}

O propósito primordial da gestão em saúde é a produção de decisões, sendo este um processo complexo que envolve crenças, motivações, interesses, intuição, conhecimentos e habilidades (MS, 2000). As crenças que os gestores da saúde possuem acerca do SUS, provavelmente, interferem em seus comportamentos diante dos processos de direção, condução e comando do sistema de saúde, como coloca Cordioli (2008). Partindo desse pressuposto, este estudo teve como objetivo principal analisar as crenças compartilhadas pelos gestores dos distritos sanitários sobre o SUS em Natal.

A partir da análise dos dados, pode-se verificar que os gestores do SUS reconhecem a grande dimensão do sistema e a necessária distribuição de suas ações em níveis de atenção hierárquica, para facilitação de sua gestão e operacionalização. Apontam ainda a perfeição teórica do modelo, que se inspira nos sistemas internacionais de países como Cuba e Canadá. Observa-se, entretanto, que os gestores reconhecem as limitações e falhas operacionais, tão reclamadas pelos usuários e denunciadas pela mídia (Melo, 2009; Polignano, 2001).

Os gestores também percebem que o SUS veio trazer a universalidade no acesso à saúde, assumindo, entretanto, que ele enfrenta barreiras operacionais, devido à falta de profissionais, às péssimas condições de infraestrutura e materiais das USFs, e à burocratização do SISREG, que, como consequência, fortalece o setor privado de saúde, que é visto como mais eficaz. Dados que corroboram o estudo de Melo (2007), onde se observou o crescimento do uso das policlínicas privadas de saúde pela classe média baixa, em detrimento do SUS. Foi verificado ainda que, conforme mostra Albuquerque (2002), os gestores reconhecem que 0 envolvimento e a participação efetiva das classes médias e altas no SUS, como pessoas mais esclarecidas, como "sujeitos de direito", pode contribuir para uma efetiva melhoria no sistema, saindo da tradição brasileira de que o serviço público é feito para pobre e que, consequentemente, pode ser de baixa qualidade.

Constatou-se, ainda, que os gestores percebem que essa problemática não ocorre pela à ausência ou limitação de dinheiro, mas em decorrência da má administração do dinheiro público destinado à saúde, dados que corroboram os achados da avaliação do SUS realizada no estado da Paraíba por Martins (2008), recursos que, poderiam ser mais diretamente fiscalizados pela população, por meio dos Conselhos Municipais de Saúde, como lembram os gestores. O bserva-se, ainda, que eles também reconhecem que a gestão do SUS e da Estratégia Saúde da Família ainda é pouco compreendida pelos seus representantes e que estes são indicados ao cargo de gestor, muitas vezes, por indicação política, tendo como critério alianças e interesses pessoais, e não por qualificação.

\section{Considerações finais}

Com base na discussão dos resultados, pôde-se perceber que, apesar de menos visíveis, os grandes entraves na realidade operacional do Sistema Único de Saúde e da Estratégia Saúde da Família, como sua porta de entrada na Atenção Básica, estão além das dificuldades visíveis nas Unidades de Saúde da Família, antes mesmo de chegar às condições limitantes de trabalho dos profissionais e da ineficácia dos serviços aos usuários. São problemas que vão desde a visão ideológica sobre os serviços de saúde, perpassando fatores políticos, deparando-se com burocracias e finalizando com barreiras que geram uma deficiência na gestão.

Sabe-se que a temática da saúde apresenta-se ampla e camuflada por princípios teóricos que não revelam sua realidade operacional falha. Compreendese também que muitos tentam modificar 0 modelo de saúde brasileiro, o criticam, e defendem a mudança das estruturas do SUS. Ao término deste trabalho, propõese, entretanto, que o problema não seja procurado nos papéis e nas formulações teóricas do modelo, e sim na operacionalização, no cotidiano do repasse de verbas, na capacitação dos profissionais, na infraestrutura dos locais de prestação de atendimento à comunidade. Trata-se de mitigar as barreiras que existem entre os dois extremos do sistema, aproximando a teoria e a prática. Trata-se de ter em mãos um sistema ideal, mas que necessita da operacionalização concreta de suas formulações, que devem sair da utopia e entrar na realidade do atendimento. Contempla-se a necessidade de instigar a população a utilizar o serviço público, lutar pela sua melhoria, incentivando a cultura de avaliação dos gastos públicos e exigências do retorno desse investimento em forma de serviços verdadeiramente eficazes na melhoria da saúde da população.

\section{Referências}

Albuquerque, F. J. B. (1999). A pontamentos para uma psicologia sócio-rural no Brasil [Resumo]. Anaisdo I Congress Note Nordeste de Psicloga (vol. 1, pp. 12-17). Salvador: UFBA.

Albuquerque, F. J. B. (2002). Social psychology and rural life in Brazil. Psicdoja: Teria ePesquisa, 18(1), 37-42. 
Albuquerque, F. J. B., \& Melo, C. F. (2010). Avaliação dos serviços públicos de saúde em duas capitais nordestinas do Brasil. Teria ePesquisa, 26(2), 323330.

Albuquerque, F. J. B., Melo, C. F., Souza Filho, F. E., \& Araújo Neto, J. L. (2011). Avaliação da estratégia saúde da família a partir das crenças dos profissionais. Estudos de Psicogia (PUCCAMP), 28(3), 363-370.

Belloni, I., Magalhães, H., \& Sousa, L. C. (2003). Metodloga de avaliação em pdíticas públicas Coleção Questões da Nossa Época. (3ํㅡㄹ. ed., vol. 75). São Paulo, SP: Cortez.

Brasil (1988). Constituição Feekal de 1988. Brasília, DF. Recuperado: 20 setembro, 2010. Disponível: http:/ / www.planalto.gov.br/ ccivil_03/ constituica o/ constitui\%C3\%A 7ao.htm.

Código de Ética Profissional do Psicólogo. (2005). São Paulo: APA. Recuperado: 20 setembro 2010. Disponível:

http:/ / www.psicologo.inf.br/ codigo_de_etica_psi cologo.asp

Cordioli, A. V. (2008). Psicterapias (3ạ ed). São Paulo, SP: Artmed.

Gomes, E. Q. (2002). A arganizaçãodos serviçs públicos na reede básica de saúde em João Pessca (Dissertação de Mestrado). Curso de Pós-Graduação em Enfermagem, Universidade Federal da Paraíba, João Pessoa, Paraíba, Brasil.

Krünger, H. (2004). Crenças compartilhadas, preconceitos e discriminações [Resumo]. Em Sociedade Brasileira de Psicologia (O rg.), Resumos de commicaçães detíficas, XXXIV Renião Anual da SociedadeBrasileira dePsicdoga Ribeirão Preto: SBP.

Lobo, T. (2001). Avaliação de processos e impactos em programas sociais: algumas questões para reflexão. Em Rico, E. M. (O rg), Avaliação de pdíticas sciais uma quesão emdbate( $3^{a}$ ed., pp. 75-85,). São Paulo, SP: Cortez.

Martins, C. (2008). Sistema ÚnicodeSaúde uma análisedas cenças dos sas representantes em muiápios nurais ma Paraíba (Dissertação de Mestrado). Curso de PósGraduação em Psicologia Social, Universidade Federal da Paraíba, João Pessoa, Paraíba, Brasil.

Melo, C. F. (2007). Avaliação do Programa Saúde da Familia (PSF) emJã̃o Pessca e Fotaleza a partir das crencas das usuánias dos PSFs dos Centros deRefêenias edas Pdidínicas (Monografia de G raduação). Curso de Graduação em Psicologia, Universidade Federal da Paraíba, João Pessoa, Paraíba, Brasil.

Melo, C. F. (2009). Avaliação da Estratééa Saúde da Familia a partir das cenças de sas profissionais (Dissertação de Mestrado). Curso de PósGraduação em Psicologia Social, Universidade
Federal da Paraíba, João Pessoa, Paraíba, Brasil.

Melo, C. F. (2011). Avaliação da Estratéǵja Saúde da Família na capital potiguar (Tese de Doutorado). Curso de Pós-Graduação em Psicologia, Universidade Federal do Rio Grande do Norte, Natal, Rio Grande do Norte, Brasil.

Ministério da Saúde [MS]. (2000). Sistema ÚnicodeSaúde (SUS): prinápios e conquistas Secretaria-executiva, Brasília, DF: Ministério da Saúde.

Ministério da Saúde [MS]. (2001). Guia prático do Programa Saúdeda Família. A SaúdeBural faz parteda Saúde da Familia? Brasília, DF: Ministério da Saúde.

Ministério da Saúde [MS]. (2004). Avaliação nomativa do Programa Saúdeda Família no Brasil: monitoramento da implantação e fumianamento das equipss de saúde da familia: 2001-2002. Série C. Projetos, Programas e Relatórios, Ministério da Saúde, Secretaria de Atenção à Saúde. Departamento de Atenção Básica. Brasília, D F: Ministério da Saúde.

Ministério da Saúde, [MS], (2006). Pdítica Nacional de Atenção Básica. Série Pactos pela Saúde. Secretária de Atenção à Saúde. D epartamento de Atenção Básica. (Vol. 4, 63 p.). Brasília, DF: Ministério da Saúde.

Negri, B. (2002). A pdítica desaúdemo Brasil nos anos 90. Série B. Textos Básicos de Saúde. Brasília: Ministério da Saúde.

Oliveira, S.F. (2006). Avaliação do Programa Saúde da Familia: uma análisea partir das cencas dos profissionais da equipedesaúdeeda pqulação assistida (D issertação de Mestrado). Curso de Pós-Graduação em Psicologia Social, Universidade Federal da Paraíba, João Pessoa, Paraíba, Brasil.

Polignano, M. V. (2001). História das políticas de saúde no Brasil: uma pequena revisão. Cademos do Intemato Rural - textos de apcio Recuperado: 20 de outubro de 2006.2 Disponível: http:/ / internatoruarl.medicina.ufmg.br/ textos.ht $\mathrm{m}$.

Rokeach, M. (1981) Crencas, atitudes e valures Rio de Janeiro, RJ: Interciência.

Silva, L. M. V., \& Formigli, V. L. A. (1994). Avaliação em saúde: limites e perspectivas. Cadkmo de Saúde Pública, Rio de Janeiro, 10(1), 80-91. Recuperado: 20 de maio de 2011. Disponível: http:/ / www.scielo.br/ pdf/ csp/ v10n1/ v10n1a09. pdf.

Tanaka, O. Y., \& Melo, C. (2000). Uma proposta de abordagem transdisciplinar para avaliação em saúde. Inteface- Commicação Saúde, Educação, 4(7), 113-118.

Tripodi, T., Fellin, P., \& Epstein, I. (1975). Avaliação de progamas Ssociais Rio de Janeiro, RJ: Livraria 
Francisco Alves.

Reedbidoem28/ 03/ 2011 Reformuladoem06/ 09/ 2011 Aprovadbem10/ 01/ 2012

Sobre os autores:

Cynthia de Freitas Melo é psicóloga. Especialista em Saúde Coletiva. Mestre em Psicologia pela Universidade Federal da Paraíba. D outoranda em Psicologia pela Universidade Federal do Rio Grande do Norte.

João Carlos Alchieri é doutor em Psicologia pela Universidade Federal do Rio Grande do Sul. Professor adjunto e bolsista produtividade (CNPq) na Universidade Federal do Rio Grande do Norte onde atua como orientador de mestrado no PPG Psicologia e mestrado e doutorado no PPG Ciências da Saúde.

João Lins de Araújo N eto é médico graduado pela Universidade Federal da Paraíba.

Contato com os autores:

Avenida Epitácio Pessoa, 735, CEP: 58030-000. João Pessoa - PB.

Email: cf.melo@yahoo.com.br 